\title{
Stride Velocity 95th Centile: Insights into Gaining Regulatory Qualification of the First Wearable-Derived Digital Endpoint for use in Duchenne Muscular Dystrophy Trials
}

\author{
Laurent Servais $^{\mathrm{a}, \mathrm{b}}$, Karl Yen ${ }^{\mathrm{c}, 1}$, Maitea Guridi ${ }^{\mathrm{c}}$, Jacek Lukawy ${ }^{\mathrm{c}, 2}$, \\ David Vissière $^{\mathrm{d}}$ and Paul Strijbos ${ }^{\mathrm{c}, *}$ \\ ${ }^{a}$ Division of Child Neurology, Centre de Références des Maladies Neuromusculaires, Department of Pediatrics, \\ University Hospital Liège and University of Liège, Liège, Belgium \\ ${ }^{\mathrm{b}}$ Muscular Dystrophy UK Oxford Neuromuscular Centre, Department of Paediatrics, University of Oxford, \\ Oxford, $U K$ \\ ${ }^{\mathrm{c}}$ F. Hoffmann-La Roche Ltd, Basel, Switzerland \\ ${ }^{\mathrm{d}}$ Sysnav SAS, Vernon, France
}

Pre-press 18 December 2021

\begin{abstract}
In 2019, stride velocity 95th centile (SV95C) became the first wearable-derived digital clinical outcome assessment (COA) qualified by the European Medicines Agency (EMA) for use as a secondary endpoint in trials for Duchenne muscular dystrophy. SV95C was approved via the EMA's qualification pathway for novel methodologies for medicine development, which is a voluntary procedure for assessing the regulatory acceptability of innovative methods used in pharmaceutical research and development. SV95C is an objective, real-world digital ambulation measure of peak performance, representing the speed of the fastest strides taken by the wearer over a recording period of 180 hours. SV95C is correlated with traditional clinic-based assessments of motor function and has greater sensitivity to clinical change over 6 months than other wearablederived stride variables, for example, median stride length or velocity. SV95C overcomes many limitations of episodic, clinic-based motor function testing, allowing the assessment of ambulation ability between clinic visits and under free-living conditions. Here we highlight considerations and challenges in developing SV95C using evidence generated by a highperformance wearable sensor. We also provide a commentary of the device's technical capabilities, which were a determining factor in the regulatory approval of SV95C. This article aims to provide insights into the methods employed, and the challenges faced, during the regulatory approval process for researchers developing new digital tools for patients with diseases that affect motor function.
\end{abstract}

Keywords: Biomarkers, clinical trial, drug approval, drug development, gait, gait analysis, movement, motor activity, neuromuscular diseases, wearable electronic devices, walking

\footnotetext{
${ }^{1}$ Current affiliation: Sanofi SA, Basel, Switzerland

${ }^{2}$ Current affiliation: Novartis International AG, Basel, Switzerland.
}

\footnotetext{
${ }^{*}$ Correspondence to: Paul Strijbos, F. Hoffmann-La Roche Ltd, Grenzacherstrasse 124, 4070 Basel, Switzerland. Tel.: +41 61688 32 35; E-mail: paul.strijbos@roche.com.
} 


\section{INTRODUCTION}

Individuals with neuromuscular diseases (NMDs) live with their condition every day, but sporadic, clinic-based motor function testing only provides a snapshot of their condition at the time of testing. Moreover, the accuracy of clinic-based testing may be compromised by factors including the patient's motivation [1] or level of fatigue [2]. Accurate detection of disease progression is vital for optimizing the outcomes of patients with NMDs and for assessing the efficacy of new treatments.

Duchenne muscular dystrophy (DMD) is a rare $\mathrm{X}$-linked, degenerative NMD, for which several promising therapeutic strategies are emerging [3]. However, inconsistent study results in recent DMD clinical development programs have raised questions about the validity of the methods used to evaluate treatment efficacy [3].

Wearables contain a variety of sensors with potential to capture real-world healthcare data at scale with minimal disruption to daily life [4]. Wearablederived clinical outcome assessments (COAs) have the potential to supplement, or even replace, clinicbased testing to provide a more accurate and complete view of the patient's condition; harnessing this potential could yield enormous benefits in clinical research and practice. Regulators recognize this opportunity, and there is a global drive to encourage the use of digital tools in regulatory decision-making [5, 6]. At the same time, the wearables market is rapidly growing; by 2022 , more than a billion wearables are predicted to be in use globally [7].

Early studies using step activity monitors suggest that wearable-derived stride parameters hold promise for monitoring disease progression in DMD $[8,9]$. Children with DMD take significantly fewer steps and spend less time at moderate and high step rates than healthy children [9]. As ambulation ability declines over time, patients experience a rapid shift from high- to low-frequency strides, with the proportion of high-frequency strides declining to zero prior to ambulation loss [8]. Changes in strides per day and the proportions of high- and low-frequency strides all correlate with 10-meter walk or run speed, which supports their potential utility in clinical studies [8].

However, regulatory endorsement is crucial for wearable-derived data to support an efficacy claim for a new treatment [10]. Acceptable outcome measures must fulfil an unmet need (improve upon currently used measures), be analytically and technically valid (demonstrating accuracy, precision, selectivity, sen- sitivity, reproducibility, and stability), be clinically valid (linked to clinically meaningful and significant changes in disease progression and correlate with disease natural history), and demonstrate content and construct validity (the measure must accurately capture movement reflecting motor function) [11, 12].

In 2019, stride velocity 95th centile (SV95C) became the first digital COA to fulfil regulatory requirements. SV95C is qualified by the European Medicines Agency (EMA) for use as a secondary endpoint in pivotal studies for DMD [13] and is under US Food and Drug Administration (FDA) review in the COA Qualification Program [14]. The EMA qualification of SV95C was based on the evaluation of magneto-inertial sensor data generated using a CEmarked class 1 medical wearable device $\left(\right.$ ActiMyo $\left.^{\circledR}\right)$. However, the EMA stipulates that SV95C may be captured by any 'valid and suitable wearable device' worn at the ankle, and the minimal technical requirement in terms of precision are defined [13]. This article describes the critical factors for successful qualification of SV95C and highlights the challenges encountered on the pathway to qualification.

\section{STRIDE VELOCITY 95TH CENTILE: CONSIDERATIONS AND CHALLENGES FOR GAINING REGULATORY APPROVAL}

\section{Ambulation parameters}

ActiMyo and the new-generation model $\left(\right.$ Syde $\left.^{\circledR}\right)$ use magneto-inertial sensors to quantify movement [15], and were specifically designed for real-world use in patients with movement disorders (Fig. 1) [16]. Although the EMA clarifies that other devices worn at the ankle may be used to capture SV95C [13], devices must meet the quality standards outlined in the EMA qualification to ensure that the data collected meet regulatory standards. Furthermore, changes to the sensor mechanism that may impact the clinical measurement properties would need to be supported by bridging data in a justified sensitive model [13]. ActiMyo is currently the only validated device suitable for capturing SV95C in clinical trials [17]. For these reasons, other devices are not discussed.

Both devices include a high-precision triaxial accelerometer, gyrometer, magnetometer, and a barometer recording data above $100 \mathrm{~Hz}$, capturing linear acceleration, angular velocity, magnetic field of movement (in all directions), and barometric 
a) ActiMyo wearable sensors (third generation)

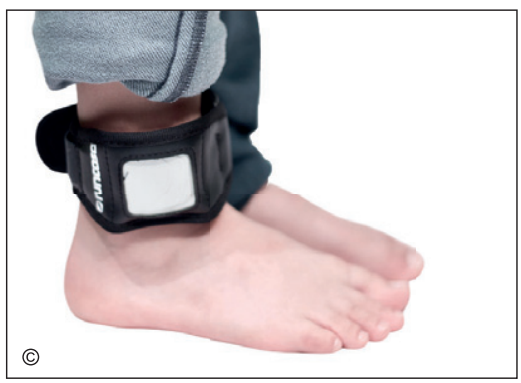

c) Syde wearable sensors (new generation)

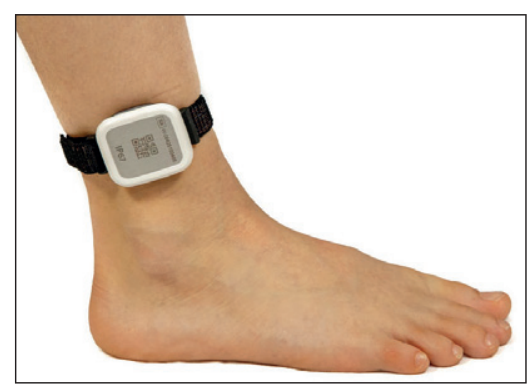

b) ActiMyo docking station (third generation)

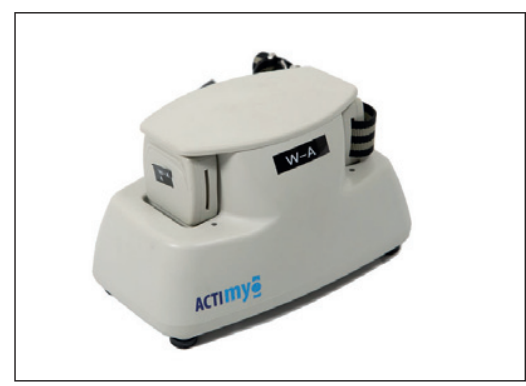

d) Syde docking station (new generation)

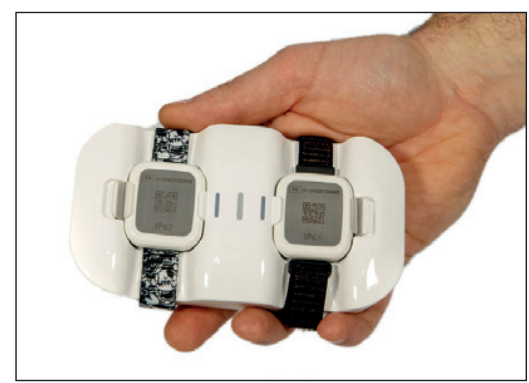

Fig. 1. Wearable magneto-inertial sensors: The third-generation ActiMyo (a and b) is succeeded by the new-generation device (Syde; $\mathrm{c}$ and d) which will be used in patients from 2021. Ambulant patients can wear a sensor strapped to each ankle, or one on the wrist and one on the ankle; sensors capture data throughout the day. The devices are transferred to a docking station overnight and the encrypted, anonymized data can be sent via a secure web cloud or stored on the internal USB drive [13]. The new-generation device (c and d) was redesigned with input from patients and healthcare professionals; it maintains the same level of sensor performance and measurement precision as its predecessor ( $a$ and $b$ ) but is one-third of the size and half the weight. The data recording station has also been modified to enable data capture for up to two weeks without internet connection or battery charging. Image a @PhotoRoom.

a)
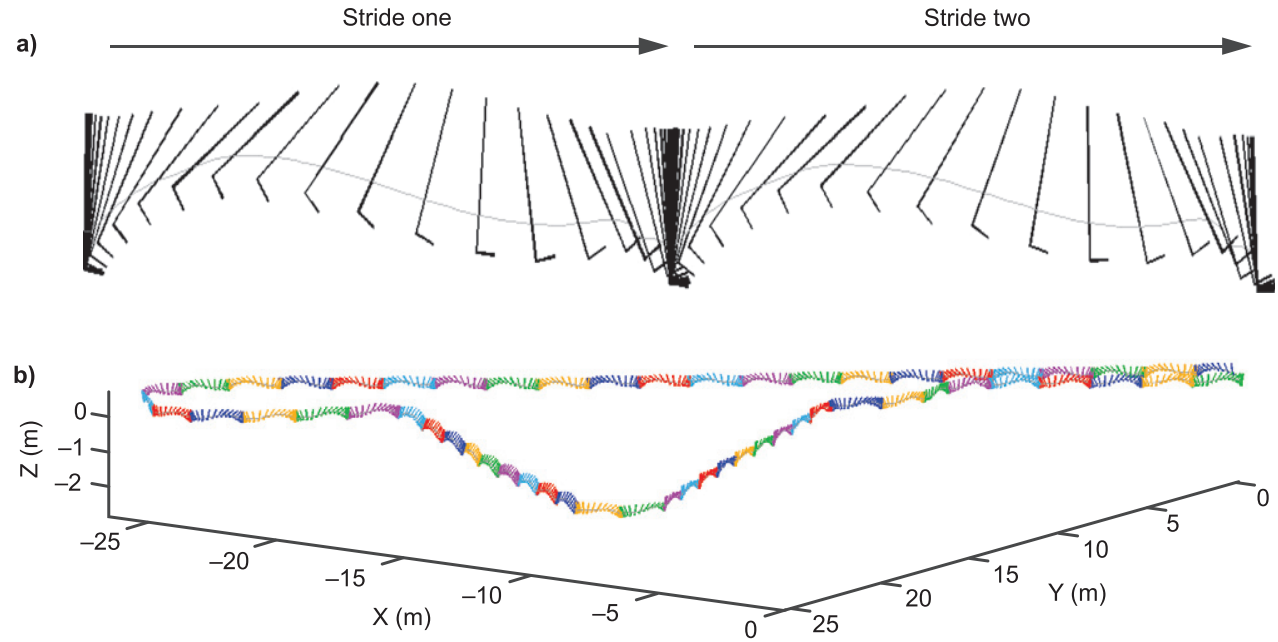

Fig. 2. Stride trajectory captured by ActiMyo. Computed trajectory of: a) two individual strides captured during ambulation; b) a circuit with stairs.

altitude [13]. The sensors were selected and individually factory-calibrated to compensate for repeatable errors (including thermal variations). Dedicated algorithms precisely and accurately capture the trajectory of the ankle, enabling high-fidelity 3D stride reconstruction, from which multiple stride parameters are derived (Fig. 2).

Three wearable-derived ambulation variables were evaluated (stride length, stride speed, and distance walked/recorded per hour) [18]. Variables were 
selected because of their clinical relevance in patients with DMD [19] and because they were robustly measurable in ambulant patients [18]. For each variable, different centiles were studied to optimize the longitudinal sensitivity to change. SV95C, reflecting the fastest strides taken, was selected for qualification based on measurement accuracy, reliability, sensitivity to change, and clinical relevance [18].

\section{Unmet need}

Timed-function tests and rating scales are predominantly used to assess motor function in NMDs, including the 6-minute walk test (6MWT), North Star Ambulatory Assessment (NSAA), and 4-stair climb [20]. In spinal muscular atrophy (SMA), the Hammersmith Functional Motor Scale Expanded and Motor Function Measure are commonly used [21, 22]. Performance on these types of measures may be influenced by patient motivation levels $[1,18]$, verbal encouragement during the test [23], and the learning effect $[18,23]$. Inter-test variability is often high [2, $24]$ and subjective rating scales may be prone to rater bias [24, 25].

EMA guidelines recommend evaluating strength as well as motor function in clinical studies for muscular dystrophies [26]. Handgrip has been proposed as a feasible and reliable measure of strength in SMA [27] and DMD [28], particularly when expressed as a percentage of predicted values for age [29], but the clinical significance of strength change remains unclear [27-29].

As part of the qualification process for SV95C, it was important to clearly describe the limitations of currently used methods for assessing motor function to provide a clear rationale for seeking qualification. In the context of NMDs, the limitations of these study endpoints can lead to variability in study results and influence clinical development programs for new treatments. For example, in DMD, phase IIb and III studies of ataluren failed to show significant improvements versus placebo in the 6MWT $[30,31]$, but benefits were observed in subgroups of patients with a baseline 6-minute walking distance (6MWD) between $300 \mathrm{~m}$ and $400 \mathrm{~m} \mathrm{[31]} \mathrm{and} \mathrm{in}$ patients with a baseline 6MWD of less than $350 \mathrm{~m}$ [30]. Similarly, eteplirsen and drisapersen showed similar mixed results in the 6MWT [32-35], although improvements in physical functioning were reported for all three treatments in long-term studies [36-39] and in patient and caregiver testimonials [39-41]. The lack of consistent efficacy data may account for discordance between the EMA and FDA; ataluren was conditionally approved by the EMA [42] but not by the FDA [43], while eteplirsen was conditionally approved by the FDA [33] but not by the EMA [44]. Drisapersen was discontinued owing to safety concerns [45]. Natural history studies have confirmed that the trajectory of longitudinal changes in the 6MWT differs depending on patient age, steroid use, and baseline 6MWD [46], although one prognostic model estimated that only $28 \%$ of variation in the 6MWT can be explained by these factors [47]. Crucially, episodic clinic-administered tests such as the 6MWT may not provide an accurate reflection of real-world ambulation ability [17].

NMDs are debilitating diseases - even small improvements in function could have a meaningful impact on the patient, but clinic-based tests may not be sensitive enough to detect small functional changes, particularly if compensatory muscle strategies are adopted in the early stages of the disease [48].

Wearables enable continuous and objective data capture from the home environment, offering a potential solution to problems associated with clinic-based testing including rater bias, performance variability due to patient motivation or verbal encouragement during the test, and the learning effect. However, identifying a wearable device with the analytical and technical validity to support regulatory decisionmaking was a key challenge in the development of SV95C.

\section{Analytical and technical validity}

The qualification process focuses on how the technology will provide clinically meaningful data, rather than the device technical specifications [49]. It is therefore important to consider early in the process what falls under the EMA remit. Device factors that could influence the reliability of the data, for example, the accuracy, precision, and clinical validity of the measure, are key considerations for qualification.

Multiple factors influence sensor accuracy, including the stability, linearity, calibration [50], noise characteristics [51], positioning, and orientation of sensor(s) [52], as well as the algorithms and methodologies used for deriving gait parameters [53, 54].

Inertial sensors (accelerometers, magnetometers, and gyroscopes) are increasingly used for ambulation assessment [52], but are reliant on machine-learning methods for activity recognition, which have variable accuracy [52]. Algorithms are typically tested in a controlled environment [55] and real-world activities 
may be wrongly interpreted (e.g., cycling as strides) [56]. Sensors do not directly measure position, but instead capture kinetic data (e.g., acceleration), and the calculated measurement values may 'drift' from the true values over time, reducing measurement accuracy [57]. Inertial sensors are prone to small errors in acceleration which are cumulative and result in 3D position drift over time [58]; magnetometers are susceptible to magnetic distortions, particularly indoors [59]; and ambient temperature fluctuations can cause gyroscope measurement drift [60].

Inertial measurement units often use fixed-threshold-based detectors to overcome drift, but these methods fail with atypical strides [56]. While SV95C may be captured by any 'valid and suitable wearable device' [13], device selection was driven by the need for validated measurement accuracy of the atypical strides characteristic of patients with NMDs.

ActiMyo stride detection is derived from the 3D trajectory of the ankle, providing a $98 \%$ stride detection success rate over 2000 atypical strides, including small or side steps [56]. The algorithms were based on an approach developed for military operations using sensors anchored to the shoe [61]. However, trajectory reconstruction is influenced by shoe rigidity and duration of contact between the foot and the ground (e.g., running vs walking) [61]. A novel patented dynamic realignment algorithm was developed for use with an ankle strap [61]. Ankle placement allows more precise trajectory reconstruction than shoe placement because stride impact is absorbed by the leg, increasing the signal to noise ratio and avoiding sensor saturation (and the resulting error accumulation) which can occur if a sensor measures a value that is larger than its dynamic range.

Movement in patients with neuromuscular diseases may be of a low amplitude, speed and acceleration - ActiMyo sensors are designed for long-term measurement stability with good signal to noise ratio, allowing accurate quantification of movement even at slow speeds [62]. Trajectory reliability and repeatability are achieved with a novel patented algorithm based on physical modeling paired with highperformance sensors. Unlike most activity trackers, ActiMyo sensors are calibrated using a rotation table and temperature-compensated to minimize sensor error and negate the need for individual a priori patient calibration [13]. Proper compensation of magnetic field variations, with multiple sequences of inertial sensor integration, permits high-fidelity 3D trajectory reconstruction and reduces magnetic field interference [13].
In healthy volunteers, ambulation parameters were captured with similar levels of accuracy to motion capture, with a peak-to-peak discrepancy rate below $2.5 \%$ [13].

Sensitive magneto-inertial sensors may capture multiple ambulation variables, but identifying the right parameters, as determined by the characteristics of the patient population, is vital for deriving meaningful motor function assessments.

\section{Clinical meaningfulness}

Step count is frequently used for real-world activity monitoring in NMDs [63]. Step count is easily captured using a pedometer, but measurement accuracy is compromised in patients with walking difficulties and at slow walking speeds [64].

Of the real-world ambulation parameters assessed, SV95C was the most sensitive indicator of clinical change in patients with DMD. After 6 months, in patients aged 7 years and above with baseline 6MWT below $450 \mathrm{~m}$, stride length 95th centile declined by $2.4 \%(p<0.05)$, median stride velocity by $4.7 \%$ $(p<0.05)$, and SV95C by $8.5 \%(p<0.01)$ [13]. In contrast, stride length increases with age and height in healthy volunteers, while SV95C remains stable over a one-year period [65]. During the qualification process, the EMA also requested evidence showing that SV95C is sensitive to positive improvements in function. This was a key point of contention because it is particularly challenging to measure positive functional changes in a disease for which there is no effective disease-modifying treatment available. Similarly, any improvement observed in a clinical trial setting would be invalid unless the experimental treatment has since been shown to be effective.

Corticosteroid treatment is associated with shortterm improvements in motor function, followed by a phase of gradual decline in DMD [66]. We have subsequently shown that SV95C is sensitive to the positive and negative functional changes associated with steroid treatment; in steroid-naïve patients, steroid initiation resulted in an $11.6 \%$ increase in SV95C after 6 months, while age-matched patients receiving stable steroid treatment experienced a $6.8 \%$ decline [67].

To demonstrate clinical meaningfulness, results must correlate to some degree with previously validated measures. All variables had moderate but significant correlations with the 6MWT and NSAA [13] (Table 1). However, a limiting factor in the qualification process was the use of the 6MWT as the gold 
Table 1

Correlation coefficients between wearable-derived gait variables recorded over 180 hours and the 6MWT and NSAA [13]

\begin{tabular}{|c|c|c|c|c|c|}
\hline \multirow[t]{2}{*}{ Gait variable } & \multirow[t]{2}{*}{$\mathrm{N}$} & \multicolumn{2}{|c|}{ 6MWT } & \multicolumn{2}{|c|}{ NSAA } \\
\hline & & Spearman coefficient & Pearson coefficient & Spearman coefficient & Pearson coefficient \\
\hline Stride length, median, $\mathrm{m}$ & 45 & $0.552^{* *}$ & $0.649^{* *}$ & $0.554^{* *}$ & $0.607^{* *}$ \\
\hline Stride length 95 th centile, $\mathrm{m}$ & 45 & $0.679^{* *}$ & $0.772^{* *}$ & $0.779^{* *}$ & $0.816^{* *}$ \\
\hline Stride velocity, median, $\mathrm{m} / \mathrm{s}$ & 45 & $0.652^{* *}$ & $0.758^{* *}$ & $0.712^{* *}$ & $0.724^{* *}$ \\
\hline Stride velocity 95 th centile, $\mathrm{m} / \mathrm{s}$ & 45 & $0.542^{* *}$ & $0.616^{* *}$ & $0.645^{* *}$ & $0.689^{* *}$ \\
\hline Distance walked/hour recorded & 45 & $0.371^{*}$ & $0.436^{* *}$ & $0.424^{* *}$ & $0.435^{* *}$ \\
\hline
\end{tabular}

${ }^{*} p<0.05$. ${ }^{* *} p<0.01$. Abbreviations: 6MWT, 6-minute walk test; NSAA, North Star Ambulatory Assessment.

standard reference point. It is inherently difficult to prove that a change in SV95C that is not detectable with the 6MWT is anything more than signal noise when there are no other, more sensitive measures available to validate the findings.

A robust assessment of the minimal clinically important difference (MCID) ensures clinical studies are adequately powered to demonstrate meaningful benefits [68]. The MCID for SV95C was calculated using the distribution-based methods described by McDonald et al. [20] as $0.1 \mathrm{~m} / \mathrm{s}$ (a relative $6.24 \%$ decline), corresponding to a $36 \mathrm{~m}$ difference in 6MWT [13] (30 $\mathrm{m}$ is the accepted 6MWT MCID in DMD trials [20]). Studies are ongoing to confirm the validity of the MCID [13].

Large sample sizes are required for studies employing the 6MWT as a primary endpoint. In the phase III ataluren study for DMD, an estimated 105 patients per treatment arm were needed to show a $30 \mathrm{~m}$ difference in 6MWT at 48 weeks with $85 \%$ power [31]. For SV95C, 14 patients per arm with DMD aged 7 years or above (with 6MWT baseline $<450 \mathrm{~m}$ ) provides sufficient statistical power (80\%) to demonstrate significant stabilization of motor function with treatment at 6 months (vs natural history) [13] indicating the improved sensitivity and reliability of SV95C versus the 6MWT. NMDs are typically rare diseases [69] and patient enrollment for studies is challenging [70]; therefore, the ability to conduct smaller, shorter clinical studies using SV95C confers a significant advantage over traditional endpoints.

\section{Usability and compliance}

Compliance is an important consideration for wearables. Data collection may be compromised if the device is difficult to use or uncomfortable to wear, or if patients avoid wearing it in public or on certain days (e.g., weekends). In patients with NMDs, muscle weakness might preclude compliance if the device is heavy. Position on the body can also influence com- pliance with wearables in children [71]. The ActiMyo ankle strap enables the user to change footwear during the day and conceal the device under clothing. The newer device is smaller and lighter than the previous model (Fig. 1), which could improve future compliance. The risks of poor compliance are mitigated through patient, caregiver, and investigator training, and the provision of clear instructions (using videos and instruction manuals).

Wearable device usage can decline over time [72]. Therefore, patient compliance was a key consideration in determining the duration of recorded data for calculating SV95C. A total of 180 hours was optimal, with good compliance and low measurement variability; $90 \%$ of patients recorded 180 hours over 1 month [13]. Mean SV95C variability was $6.38 \%$ at 50 hours of recording (standard deviation [SD], $2.60 \%$ ); this reduced to $4.41 \%$ (SD, 2.33\%) at 180 hours [13]. Data-processing software flags a warning if step count is abnormally low or sensors fail [13]. Data are deemed missing if under 50 hours are recorded over 1 month [13]. Evaluating the possible causes of measurement variability and mathematically defining the optimum recording period were contributing factors for success in the qualification process for SV95C.

\section{Data privacy}

Regulation of ownership and responsibility for wearable-generated data are lacking [73], and wearables may be vulnerable to security breaches if security is not prioritized in development. Wearer concerns regarding data privacy could also reduce compliance. A risk analysis was conducted to identify, address, and mitigate risks relating to data integrity, availability, and confidentiality [13]. The EMA deemed the risk level acceptable for several reasons: ActiMyo records motion, not global positioning [13]; no personal information is associated with data capture [13]; motion data are stored in a binary format 
interpretable only with extraction software and communication channels are encrypted [13]; and data are identified using a unique patient identification code linked to the patient's personal information at the clinical center and accessible only to the researcher [13].

\section{DISCUSSION}

\section{Impact of SV95C on DMD clinical studies}

SV95C is the first regulatory-approved, digital COA qualified for use as a secondary endpoint in pivotal DMD studies. This landmark qualification enables researchers to continuously track real-world patient ambulation in clinical studies, potentially transforming the development of new treatments in an area of significant unmet need.

As highlighted by the EMA, SV95C has potential as a primary endpoint in DMD trials with further supportive data [13]. Strengthening the correlation between SV95C and functional tests with longer follow-up and additional patients, expanding normative data, and further justification of the MCID are recommended [13]. Research is ongoing to support primary endpoint qualification.

As a primary endpoint, the sensitivity of SV95C should allow shorter studies enrolling fewer patients, reduce patient exposure to experimental treatments, and lower patient burden by enabling less frequent, shorter clinic visits than traditional measures. Ultimately, this work will help bring effective treatments to patients with DMD, and potentially other NMDs, sooner than previously possible. Confounding factors and the associated uncertainty of traditional study endpoints have hindered development of DMD treatments; SV95C overcomes these limitations, providing a robust rationale for adoption.

\section{Broader applications of wearable magneto-inertial sensors}

Magneto-inertial sensors offer the opportunity to provide a gold-standard measure of disease progression for use in clinical development and clinical practice, allowing continuous movement capture and assessment of natural functional ability under freeliving conditions in the real world. Sensors are being used to evaluate other important functional outcomes, including falls, stair-climbing ability, and other ambulation parameters [13], and could provide insights into the quality of movement as well as the quantity, potentially offering more granularity than traditional motor function assessments. Indeed, in the final qualification opinion, the EMA strongly encouraged further research into other outcomes, including quality of walking [13]. Several variables for upper-limb function have been identified using sensors strapped to the wrist, which could expand use to non-ambulant patients [62].

Continuous capture and quantification of movement could yield insights into many diseases. Work is ongoing to identify disease-specific measures in spinal muscular atrophy $[21,22]$, facioscapulohumeral muscular dystrophy [74], centronuclear myopathy [75], and amyotrophic lateral sclerosis. However, an integrated value of physical activity, combining lower- and upper-limb movements in patients with NMDs remains to be validated.

Beyond the neuromuscular field, ActiMyo is currently being evaluated in Angelman syndrome, Parkinson's disease, and multiple sclerosis. The ability to quantify physical activity has broad application in a range of contexts. The $6 \mathrm{MWT}$ is a prognostic indicator in heart failure [76] and chronic obstructive pulmonary disorder [77]. Physical activity is an indicator of quality of life in elderly patients [78], patients with cancer receiving chemotherapy [79], and patients with eye diseases such as glaucoma [80]. Continuous activity tracking is also valuable in patients recovering from bone fractures [81].

\section{Digital tools could improve clinical research and patient outcomes}

The accuracy of the data derived from commercialor consumer-grade devices may vary depending on the device used [82], the placement of the device [83], and the mobility of the wearer [84]. These concerns regarding accuracy and the lack of regulatory support have limited the incorporation of wearables into clinical trials $[85,86]$.

However, the development of clinically validated, high-performance wearable technologies provides an exceptional opportunity to improve the efficiency of clinical and real-world studies and could yield insights into disease progression to inform clinical practice in the future. Other promising efforts are ongoing, for example, the MOBILISE-D initiative aims to implement and validate a panel of regulatory-grade, real-world digital mobility outcomes for patients with mobility difficulties [87]. Although a global mobility assessment would have broader utility than SV95C, developing a validated 
measure across multiple diseases presents complex challenges. Disease course and clinical characteristics differ considerably across different patient populations [88] and the current EMA qualification process may need to evolve to accommodate a measure developed across multiple diseases using a common approach.

Another promising effort is the KineDMD study, which is using high-resolution wearable sensors to track full-skeleton movements and derive a complete picture of full-body behavioral capacity over a 12month period in patients with DMD [89]. The data collected will be used to develop novel clinical endpoints in the future [90].

Beyond the assessment of motor function, wearables can now quantify a range of different clinical parameters, from vital signs [91] to sleep [92], which could yield deeper insights into many other diseases in the future.

Development of these types of digital COAs could not only permit shorter, smaller studies, but also enable earlier go/no-go decisions on experimental treatments to reduce the risk of late-stage study failures and bring effective treatments to patients faster and more affordably than previously possible.

However, the process of developing and validating a digital endpoint is by no means easy or quick. We strongly advocate scientific rigor and comprehensive analytical, technical, and clinical validation of digital technologies used in clinical studies. This requires significant investment and resource, and is dependent upon successful collaborations between data scientists, device and algorithm engineers, clinical drug development teams, and regulators.

The qualification process itself is lengthy, with a duration of at least 160 days for qualification advice and 250 days for the qualification opinion [93]. The EMA encourages applicants to engage with them early in the process and to seek qualification from the FDA in parallel [94, 95], enabling support of global clinical development programs. It is worth noting that there are fundamental differences between the EMA and FDA programs; the EMA is primarily focused on the metric properties of the outcome, whereas the FDA is primarily interested in the clinical validity.

As the first successful applicants to complete the EMA process, we did encounter some initial difficulties. This is unsurprising given how rapidly technology has evolved over the last few years. The EMA is continuing to evolve their process and has since published new guidance to support applicants in the qualification process [49].

We hope our work provides a rationale for increased investment in the clinical validation of wearables. While the returns may not be immediate, in the future, the volume and breadth of data captured could generate actionable insights to improve patient outcomes across the care continuum, for example, enabling the discovery of novel diagnostic, predictive, and prognostic biomarkers (Fig. 3). Wearables could enable patients living in remote areas to participate in clinical studies, and could reveal insights into

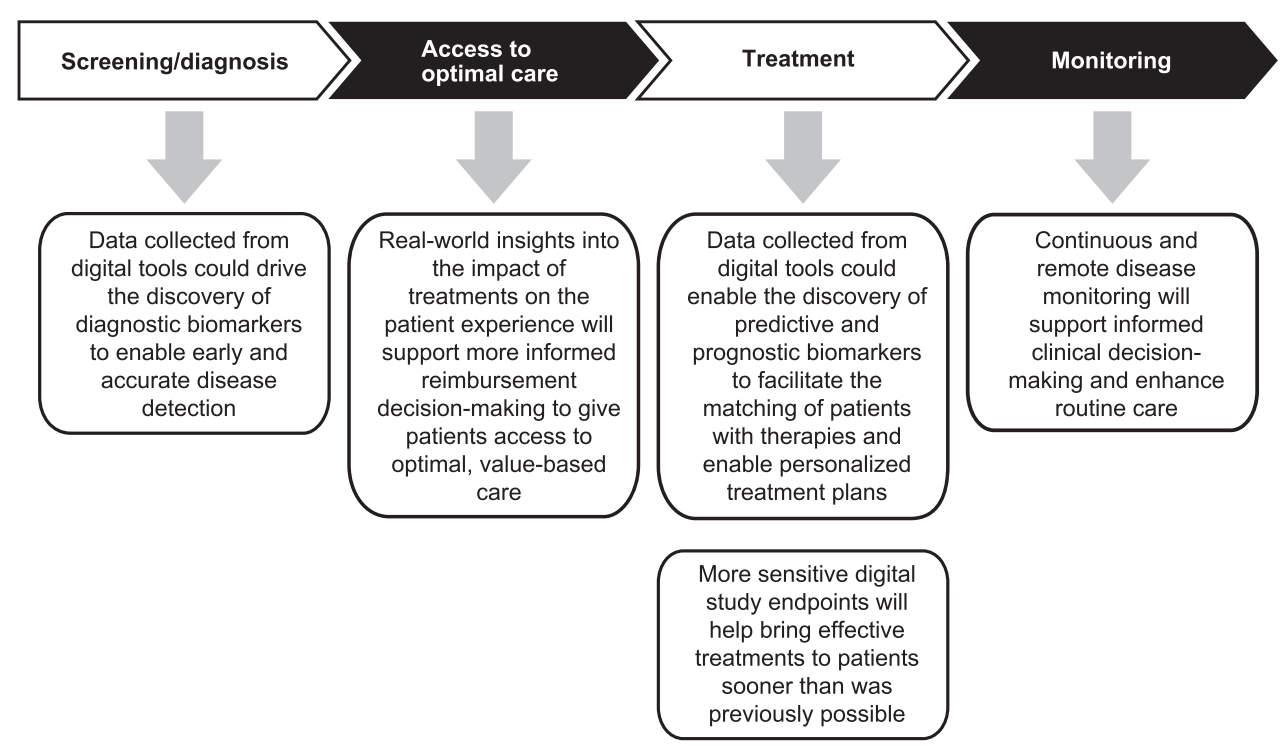

Fig. 3. Potential impact of digital data on patient care in the future. Data collected from digital tools will enable the generation of actionable insights that could improve patient outcomes across the care continuum. 
the real-world patient experience, enabling regulators, health technology assessment bodies, and payers to make informed decisions on the safety, efficacy, and value of new medicines. In the advent of genetic therapies for inherited NMDs, digital tools will be invaluable for assessing the durability of singledose treatments. For antisense therapies requiring repeat dosing, digital tools could enable early detection of non-responders to minimize patient exposure to costly ineffective treatments. For patients with NMDs, use of digital tools could provide a higher standard of care, access to value-based treatments with fewer clinic visits, and less need for onerous and repetitive performance assessments.

\section{CONCLUDING REMARKS}

Advances in sensor technology, cloud-based storage and computing, and the development of novel methods for accurate and precise trajectory reconstruction present new opportunities to transform drug development in NMDs, but regulatory qualification is essential for realizing this potential.

In a new era of technological innovation in medicine, EMA recognition of SV95C represents a landmark in the development of disease-specific mobility assessments using wearables. The ability to continuously measure function with high accuracy and precision, during free-living conditions in the real world, could fundamentally change the way we develop treatments and care for patients with many chronic diseases. We hope our work provides valuable insight and a framework that will facilitate the future development of wearable-derived COAs.

\section{FUNDING}

Funding for the article was provided by F. Hoffmann-La Roche Ltd, Basel, Switzerland.

\section{ACKNOWLEDGMENTS}

Under the direction of the authors and funded by F. Hoffmann-La Roche Ltd, Dr K. Dodd, of Oxford PharmaGenesis, Cardiff, UK, provided writing assistance for this publication. Editorial assistance in formatting, proofreading, copy-editing, and fact checking was also provided by Oxford PharmaGenesis. The authors acknowledge the work of Anne-Gaelle Le Moing, Teresa Gidaro, Andreea Seferian, Amélie Moraux, Charlotte Lilien, and Erwan Gasnier on the development of clinical vari- ables using ActiMyo, including the stride velocity 95th centile.

\section{AUTHOR CONTRIBUTIONS}

All authors made substantial contributions to the conception or design of the work, participated in drafting and revisions, and provided final approval of the article.

\section{CONFLICTS OF INTERESTS}

Laurent Servais is a member of scientific advisory boards for Novartis Gene Therapies (formerly AveXis), Biogen, Biophytis, Cytokinetics, Dynacure, F. Hoffmann-La Roche Ltd, GeneTx Biotherapeutics, REGENXBIO, Santhera, and Sarepta and has consulted for Pfizer and Affinia. Laurent Servais conducts research funded by Novartis Gene Therapies (formerly AveXis), Biogen, and F. Hoffmann-La Roche Ltd and holds a part on the patent WO201712 9890A1 with no financial interest. Karl Yen is a former employee of F. Hoffmann-La Roche Ltd. Jacek Lukawy is a former employee of F. Hoffmann-La Roche Ltd and holds stock options. Maitea Guridi and Paul Strijbos are employees of, and hold stocks in, F. Hoffmann-La Roche Ltd. David Vissière is the founder and president of the ActiMyo/Syde manufacturer Sysnav.

\section{DATASETS}

No datasets were generated or analyzed during the current study.

\section{REFERENCES}

[1] Alfano L, Lowes L, Berry K, Flanigan K, Cripe L, Mendell J. Role of motivation on performance of the 6-minute walk test in boys with Duchenne muscular dystrophy. Dev Med Child Neurol. 2015;57(S5):57-8.

[2] Prahm KP, Witting N, Vissing J. Decreased variability of the 6-minute walk test by heart rate correction in patients with neuromuscular disease. PloS One. 2014;9(12):e114273.

[3] Grages SM, Bell M, Berlau DJ. New and emerging pharmacotherapy for duchenne muscular dystrophy: A focus on synthetic therapeutics. Expert Opin Pharmacother. 2020; 21(7):841-51.

[4] Dobkin BH, Dorsch A. The promise of mHealth: Daily activity monitoring and outcome assessments by wearable sensors. Neurorehabil Neural Repair. 2011;25(9):788-98.

[5] US Food and Drug Administration. The long run is now: How FDA is advancing digital tools for medical product development. (2018) Available at: https://www.fda.gov/ news-events/speeches-fda-officials/long-run-now-how-fda -advancing-digital-tools-medical-product-development-10 252018. (Accessed October 25, 2020). 
[6] European Medicines Agency. Regulatory Science to 2025 Reference material. (2018) Available at: https://www.ema. europa.eu/en/documents/other/reference-material-multista keholder-workshop-launch-consultation-european-medici nes-agency-ema-human_en.pdf. (Accessed October 25, 2021).

[7] Statista. Connected wearable devices worldwide 20162021. (2018) Available at: https://www.statista.com/statis tics/487291/global-connected-wearable-devices/.

(Accessed October 25, 2021).

[8] Fowler EG, Staudt LA, Heberer KR, Sienko SE, Buckon $\mathrm{CE}$, Bagley AM, et al. Longitudinal community walking activity in Duchenne muscular dystrophy. Muscle \& Nerve. 2018;57(3):401-6.

[9] McDonald CM, Widman LM, Walsh DD, Walsh SA, Abresch RT. Use of step activity monitoring for continuous physical activity assessment in boys with Duchenne muscular dystrophy. Archives of Physical Medicine and Rehabilitation. 2005;86(4):802-8.

[10] Izmailova ES, Wagner JA, Perakslis ED. Wearable devices in clinical trials: Hype and hypothesis. Clin Pharmacol Ther. 2018;104(1):42-52.

[11] Hobart JC, Lamping DL, Thompson AJ. Evaluating neurological outcome measures: The bare essentials. J Neurol Neurosurg Psychiatry. 1996;60(2):127-30.

[12] Louie C, D'Agostino EN, Han D, Ryken TC. Determining an appropriate outcome measure in neurosurgical research: Investigating meaningful, valid, and practical metrics. Cureus. 2019;11(9):e5610.

[13] European Medicines Agency. Qualification opinion on stride velocity 95th centile as a secondary endpoint in Duchenne muscular dystrophy measured by a valid and suitable wearable device. (2019) Available at: https://www. ema.europa.eu/en/documents/scientific-guideline/quali fication-opinion-stride-velocity-95th-centile-secondaryendpoint-duchenne-muscular-dystrophy_en.pdf. (Accessed October 25, 2021).

[14] US Food and Drug Administration. Clinical Outcome Assessments (COA) Qualification Program. Letter of Intent: ActiMyo. Available at: https://www.fda.gov/media/119254/ download. (Accessed October 25, 2021).

[15] Lilien C, Gasnier E, Gidaro T, Seferian A, Grelet M, Vissière $\mathrm{D}$, et al. Home-based monitor for gait and activity analysis. J Vis Exp. 2019(150).

[16] Institute of Myology. ActiMyo. Available at: https://www. institut-myologie.org/imotion/actimyo/?lang=en. (Accessed October 25, 2021).

[17] Servais L, Camino E, Clement A, McDonald CM, Lukawy J, Lowes LP, et al. First Regulatory Qualification of a Novel Digital Endpoint in Duchenne Muscular Dystrophy: A Multi-Stakeholder Perspective on the Impact for Patients and for Drug Development in Neuromuscular Diseases. Digital Biomarkers. 2021;5(2):183-90.

[18] Haberkamp M, Moseley J, Athanasiou D, de Andres-Trelles F, Elferink A, Rosa MM, et al. European regulators' views on a wearable-derived performance measurement of Ambulation for Duchenne muscular dystrophy regulatory trials. Neuromuscul Disord. 2019;29(7):514-6.

[19] Goudriaan M, Van den Hauwe M, Dekeerle J, Verhelst L, Molenaers G, Goemans N, et al. Gait deviations in Duchenne muscular dystrophy-Part 1. A systematic review. Gait \& posture. 2018;62:247-61.

[20] McDonald CM, Henricson EK, Abresch RT, Florence J, Eagle M, Gappmaier E, et al. The 6-minute walk test and other clinical endpoints in duchenne muscular dystro- phy: Reliability, concurrent validity, and minimal clinically important differences from a multicenter study. Muscle Nerve. 2013;48(3):357-68.

[21] Chabanon A, Seferian AM, Daron A, Péréon Y, Cances $\mathrm{C}$, Vuillerot $\mathrm{C}$, et al. Prospective and longitudinal natural history study of patients with Type 2 and 3 spinal muscular atrophy: Baseline data NatHis-SMA study. PLoS One. 2018;13(7):e0201004.

[22] Annoussamy M, Seferian AM, Daron A, Péréon Y, Cances $C$, Vuillerot $C$, et al. Natural history of Type 2 and 3 spinal muscular atrophy: 2-year NatHis-SMA study. Ann Clin Transl Neurol. 2021;8(8):359-73.

[23] Morales Mestre N, Audag N, Caty G, Reychler G. Learning and encouragement effects on six-minute walking test in children. J Pediatr. 2018;198:98-103.

[24] Reilmann R, Schubert R. Chapter 18 - Motor outcome measures in Huntington disease clinical trials. In: Feigin AS, Anderson KE, editors. Handbook of Clinical Neurology. 144: Elsevier; 2017. pp. 209-25.

[25] Dorsey ER, Papapetropoulos S, Xiong M, Kieburtz K. The first frontier: Digital biomarkers for neurodegenerative disorders. Digital Biomarkers. 2017;1(1):6-13.

[26] European Medicines Agency. Guideline on the clinical investigation of medicinal products for the treatment of Duchenne and Becker muscular dystrophy. (2015) Available at: https://www.ema.europa.eu/en/documents/scientificguideline/guideline-clinical-investigation-medicinalproducts-treatment-duchenne-becker-musculardystrophy_en.pdf. (Accessed October 25, 2021)

[27] Seferian AM, Moraux A, Canal A, Decostre V, Diebate O, Le Moing AG, et al. Upper limb evaluation and one-year follow up of non-ambulant patients with spinal muscular atrophy: An observational multicenter trial. PLoS One. 2015;10(4):e0121799.

[28] Seferian AM, Moraux A, Annoussamy M, Canal A, Decostre V, Diebate O, et al. Upper limb strength and function changes during a one-year follow-up in non-ambulant patients with Duchenne Muscular Dystrophy: An observational multicenter trial. PLoS One. 2015;10(2):e0113999.

[29] Hogrel J-Y, Decostre V, Ledoux I, de Antonio M, Niks EH, de Groot I, et al. Normalized grip strength is a sensitive outcome measure through all stages of Duchenne muscular dystrophy. J Neurol. 2020;267(7):2022-8.

[30] Bushby K, Finkel R, Wong B, Barohn R, Campbell C, Comi $\mathrm{GP}$, et al. Ataluren treatment of patients with nonsense mutation dystrophinopathy. Muscle Nerve. 2014;50(4):477-87.

[31] McDonald CM, Campbell C, Torricelli RE, Finkel RS, Flanigan KM, Goemans N, et al. Ataluren in patients with nonsense mutation Duchenne muscular dystrophy (ACT DMD): A multicentre, randomised, double-blind, placebocontrolled, phase 3 trial. Lancet. 2017;390(10101):148998.

[32] Mendell JR, Rodino-Klapac LR, Sahenk Z, Roush K, Bird L, Lowes LP, et al. Eteplirsen for the treatment of Duchenne muscular dystrophy. Ann Neurol. 2013;74(5):637-47.

[33] Aartsma-Rus A, Krieg AM. FDA approves eteplirsen for Duchenne muscular dystrophy: The next chapter in the eteplirsen saga. Nucleic Acid Ther. 2017;27(1):1-3.

[34] European Medicines Agency. Assessment report for Kyndrisa. (2016) Available at: https://www.ema.europa.eu/ documents/withdrawal-report/withdrawal-assessment-repo rt-kyndrisa_en.pdf. (Accessed October 25, 2021).

[35] Goemans N, Mercuri E, Belousova E, Komaki H, Dubrovsky A, McDonald CM, et al. A randomized placebocontrolled phase 3 trial of an antisense oligonucleotide, 
drisapersen, in Duchenne muscular dystrophy. Neuromuscul Disord. 2018;28(1):4-15.

[36] Iff J, Gerrits C, Sajeev G, Signorovitch J, Tuttle E, Birk E, et al. Eteplirsen delays time to loss of ambulation in patients with Duchenne muscular dystrophy compared with patients receiving standard of care. Muscular Dystrophy Association Clinical and Scientific Conference; 13-17 April 2019; Orlando, USA.

[37] Charleston JS, Schnell FJ, Dworzak J, Donoghue C, Lewis $\mathrm{S}$, Chen L, et al. Eteplirsen treatment for Duchenne muscular dystrophy: Exon skipping and dystrophin production. Neurology. 2018;90(24):e2146-e54.

[38] Delage A, Bucella F, Desguerre I, Muntoni F, Osorio AN, Tulinius M, et al. Effect of Ataluren on Age at Loss of Ambulation in Nonsense Mutation Duchenne Muscular Dystrophy: Observational Data from the STRIDE Registry (P1.6-067). Neurology. 2019;92(15 Supplement):P1.6-067.

[39] New drug assessment of drisapersen for Duchenne Muscular Dystrophy. US Food and Drug Administration Available at: https://www.fda.gov/media/95912/download.(2015). (Accessed October 25, 2021).

[40] US Food and Drug Administration. New drug assessment of eteplirsen for Duchenne Muscular Dystrophy (redacted). Available at: https://www.accessdata.fda.gov/drugsatfda_ docs/nda/2016/206488Orig1 s000SumR.pdf.(2016). (Accessed October 25, 2021).

[41] US Food and Drug Administration. New drug assessment of ataluren for Duchenne Muscular Dystrophy. (2017) Available at: https://www.fda.gov/media/109528/download. (Accessed October 25, 2021).

[42] European Medicines Agency. Translarna. Available at: https://www.ema.europa.eu/en/documents/procedural-step s-after/translarna-epar-procedural-steps-taken-scientific-in formation-after-authorisation_en.pdf. (Accessed October 25, 2021).

[43] US Food and Drug Administration. New drug application. Ataluren. (2017) Available at: https://www.fda.gov/do wnloads/advisorycommittees/committeesmeetingmateri als/drugs/peripheralandcentralnervoussystemdrugsadvisory committee/ucm577349.pdf. (Accessed October 25, 2021).

[44] Aartsma-Rus A, Krieg A, Goemans N. A sequel to the eteplirsen saga: Eteplirsen is approved in the United States but was not approved in Europe. Nucleic Acid Ther. 2018; 29(1):13-5.

[45] Hilhorst N, Spanoudi-Kitrimi I, Goemans N, Morren MA. Injection site reactions after long-term subcutaneous delivery of drisapersen: A retrospective study. Eur J Pediatr. 2019;178(2):253-8.

[46] Pane M, Mazzone ES, Sivo S, Sormani MP, Messina S, $\mathrm{D}^{\prime}$ Amico A, et al. Long Term Natural History Data in Ambulant Boys with Duchenne Muscular Dystrophy: 36-Month Changes. PLOS ONE. 2014;9(10):e108205.

[47] Goemans N, vanden Hauwe M, Signorovitch J, Swallow E, Song J, Collaborative Trajectory Analysis P. Individualized Prediction of Changes in 6-Minute Walk Distance for Patients with Duchenne Muscular Dystrophy. PLOS ONE. 2016;11(10):e0164684.

[48] Doglio L, Pavan E, Pernigotti I, Petralia P, Frigo C, Minetti C. Early signs of gait deviation in Duchenne muscular dystrophy. Eur J Phys Rehabil Med. 2011;47(4):587-94.

[49] European Medicines Agency. Questions and answers: Qualification of digital technology-based methodologies to support approval of medicinal products. (2020) Available at: https://www.ema.europa.eu/en/documents/other/questions- answers-qualification-digital-technology-based-methodol ogies-support-approval-medicinal_en.pdf (Accessed October 25, 2021).

[50] Aydemir GA, SaranlıA. Characterization and calibration of MEMS inertial sensors for state and parameter estimation applications. Measurement. 2012;45(5):1210-25.

[51] Cereatti A, Trojaniello D, Della Croce U. Accurately measuring human movement using magneto-inertial sensors: Techniques and challenges. 2015 IEEE International Symposium on Inertial Sensors and Systems (ISISS) Proceedings. 2015:1-4.

[52] Attal F, Mohammed S, Dedabrishvili M, Chamroukhi F, Oukhellou L, Amirat Y. Physical Human Activity Recognition Using Wearable Sensors. Sensors (Basel). 2015;15(12): 31314-38

[53] Caldas R, Mundt M, Potthast W, de Lima Neto FB, Markert B. A systematic review of gait analysis methods based on inertial sensors and adaptive algorithms. Gait Posture. 2017;57:204-10.

[54] Yang S, Li Q. Inertial sensor-based methods in walking speed estimation: A systematic review. Sensors (Basel). 2012;12(5):6102-16.

[55] Vienne A, Barrois RP, Buffat S, Ricard D, Vidal P-P. Inertial sensors to assess gait quality in patients with neurological disorders: A systematic review of technical and analytical challenges. Front Psychol. 2017;8:817.

[56] Beaufils B, Chazal F, Grelet M, Michel B. Robust stride detector from ankle-mounted inertial sensors for pedestrian navigation and activity recognition with machine learning approaches. Sensors (Basel). 2019;19(20):4491.

[57] Takeda R, Lisco G, Fujisawa T, Gastaldi L, Tohyama H, Tadano S. Drift Removal for Improving the Accuracy of Gait Parameters Using Wearable Sensor Systems. Sensors. 2014;14(12):23230-47.

[58] Ahmadi A, Destelle F, Unzueta L, Monaghan DS, Linaza MT, Moran K, et al. 3D human gait reconstruction and monitoring using body-worn inertial sensors and kinematic modeling. IEEE Sens J. 2016;16(24):8823-31.

[59] Ilyas M, Cho K, Baeg S-H, Park S. Drift reduction in pedestrian navigation system by exploiting motion constraints and magnetic field. Sensors (Basel). 2016;16(9): 1455.

[60] Shiau JK, Ma DM, Huang CX, Chang MY. MEMS gyroscope null drift and compensation based on neural network. Advanced Materials Research. 2011;255:2077-81.

[61] Vissiere D, Hillion M, Dorveaux E, Augustin J, Grelet $\mathrm{M}$, inventors; SYSNAV, assignee. Method for estimating the movement of a pedestrian. United States patent US20180292230A1. 2019-01-25.

[62] Le Moing AG, Seferian AM, Moraux A, Annoussamy M, Dorveaux E, Gasnier E, et al. A movement monitor based on magneto-inertial sensors for non-ambulant patients with Duchenne muscular dystrophy: A pilot study in controlled environment. PLoS One. 2016;11(6):e0156696.

[63] Jimenez-Moreno AC, Newman J, Charman SJ, Catt M, Trenell MI, Gorman GS, et al. Measuring habitual physical activity in neuromuscular disorders: A systematic review. J Neuromuscul Dis. 2017;4:25-52.

[64] Elsworth C, Dawes H, Winward C, Howells K, Collett J, Dennis A, et al. Pedometer step counts in individuals with neurological conditions. Clin Rehabil. 2009;23(2):171-5.

[65] Poleur M, Ulinici A, Daron A, Schneider O, Farra FD, Demonceau $\mathrm{M}$, et al. Normative data on spontaneous stride velocity, stride length, and walking activity in a 
non-controlled environment. Orphanet J Rare Dis. 2021; 16(1):318.

[66] Griggs RC, Herr BE, Reha A, Elfring G, Atkinson L, Cwik $\mathrm{V}$, et al. Corticosteroids in Duchenne muscular dystrophy: Major variations in practice. Muscle Nerve. 2013;48(1): 27-31.

[67] Servais L, Gidaro T, Seferian A, Gasnier E, Daron A, Ulinici A, et al. P. 191Maximal stride velocity detects positive and negative changes over 6-month-time period in ambulant patients with Duchenne muscular dystrophy. Neuromuscul Disord. 2019;29:S105.

[68] Neely JG, Karni RJ, Engel SH, Fraley PL, Nussenbaum B, Paniello RC. Practical guides to understanding sample size and minimal clinically important difference (MCID). Otolaryngol Head Neck Surg. 2007;136(1):14-8.

[69] Emery AEH. Population frequencies of inherited neuromuscular diseases-A world survey. Neuromuscul Disord. 1991;1(1):19-29.

[70] El Mouelhi M. Drug development and challenges for neuromuscular clinical trials. J Mol Neurosci. 2016;58(3): 374-8.

[71] Schaefer SE, Van Loan M, German JB. A feasibility study of wearable activity monitors for pre-adolescent school-age children. Prev Chronic Dis. 2014;11:E85.

[72] Hermsen S, Moons J, Kerkhof P, Wiekens C, De Groot M. Determinants for Sustained Use of an Activity Tracker: Observational Study. JMIR Mhealth Uhealth. 2017;5(10): e164.

[73] Kostkova P, Brewer H, de Lusignan S, Fottrell E, Goldacre B, Hart G, et al. Who Owns the Data? Open Data for Healthcare. Front Public Health. 2016;4:7.

[74] Gidaro T, Gasnier E, Annoussamy M, Vissing J, Attarian S, Mozaffar T, et al. Home-based gait analysis as an exploratory endpoint during a multicenter phase 1 trial in limb girdle muscular dystrophy type R2 and facioscapulohumeral muscular dystrophy. Muscle \& Nerve. Forthcoming 2021.

[75] Annoussamy M, Gasnier E, Baets J, Schara U, Grangé A, Lilien C, et al. Feasibility and baseline values of continuous movement measurement in patients with centronuclear myopathy by using ActiMyo. Neuromuscular Disorders. 2019;29:S107.

[76] Rubim VS, Drumond Neto C, Romeo JL, Montera MW. Prognostic value of the Six-Minute Walk Test in heart failure. Arq Bras Cardiol. 2006;86(2):120-5.

[77] Takigawa N, Tada A, Soda R, Date H, Yamashita M, Endo S, et al. Distance and oxygen desaturation in 6-min walk test predict prognosis in COPD patients. Respir Med. 2007;101(3):561-7.

[78] Puciato D, Borysiuk Z, Rozpara M. Quality of life and physical activity in an older working-age population. Clin Interv Aging. 2017;12:1627-34.

[79] Kripp M, Heußer AL, Belle S, Gerhardt A, Merx K, Hofmann WK, et al. Does physical activity improve quality of life in cancer patients undergoing chemotherapy? Oncol Res Treat. 2015;38(5):230-6.

[80] Huang W, Gao K, Liu Y, Liang M, Zhang X. The adverse impact of glaucoma on psychological function and daily physical activity. J Ophthalmol. 2020;2020:9606420.

[81] Schmal H, Holsgaard-Larsen A, Izadpanah K, Brønd JC, Madsen CF, Lauritsen J. Validation of activity tracking procedures in elderly patients after operative treatment of proximal femur fractures. Rehabil Res Pract. 2018;2018: 3521271 .
[82] Case MA, Burwick HA, Volpp KG, Patel MS. Accuracy of Smartphone Applications and Wearable Devices for Tracking Physical Activity Data. JAMA. 2015;313(6):625-6.

[83] Hildebrand M, Van Hees VT, Hansen BH, Ekelund U. Age group comparability of raw accelerometer output from wrist- and hip-worn monitors. Med Sci Sports Exerc. 2014;46(9):1816-24.

[84] Straiton N, Alharbi M, Bauman A, Neubeck L, Gullick J, Bhindi $\mathrm{R}$, et al. The validity and reliability of consumergrade activity trackers in older, community-dwelling adults: A systematic review. Maturitas. 2018;112:85-93.

[85] Izmailova ES, Wagner JA, Ammour N, Amondikar N, BellVlasov A, Berman S, et al. Remote Digital Monitoring for Medical Product Development. Clinical and Translational Science. 2021;14(1):94-101.

[86] Cox SM, Lane A, Volchenboum SL. Use of Wearable, Mobile, and Sensor Technology in Cancer Clinical Trials. JCO Clinical Cancer Informatics. 2018(2):1-11.

[87] Mobilise-D. Connecting digital mobility assessment to clinical outcomes for regulatory and clinical endorsement. Available at: https://www.mobilise-d.eu/. (Accessed October 25, 2021).

[88] Rochester L, Mazzà C, Mueller A, Caulfield B, McCarthy $\mathrm{M}$, Becker C, et al. A roadmap to inform development, validation and approval of digital mobility outcomes: The Mobilise-D approach. Digital Biomarkers. 2020;4(1): 13-27.

[89] Ricotti V, Kadirvelu B, Selby V, Voit T, Faisal A. Towards high-resolution clinical digital biomarkers for Duchenne muscular dystrophy. Neuromuscul Disord. 2019;29:S108.

[90] Ricotti V, Kadirvelu B, Auepanwiriyakul C, Zeng S, Selby V, Voit T, et al. P. 205Daily life digital biomarkers for longitudinal monitoring of Duchenne muscular dystrophy with wearable sensors. Neuromuscular Disorders. 2019;29: S109.

[91] Suresh Kumar V, Krishnamoorthi C. Development of electrical transduction based wearable tactile sensors for human vital signs monitor: Fundamentals, methodologies and applications. Sensors and Actuators A: Physical. 2021;321: 112582.

[92] Guillodo E, Lemey C, Simonnet M, Walter M, Baca-García E, Masetti V, et al. Clinical applications of mobile health wearable-based sleep monitoring: Systematic review. JMIR mHealth and uHealth. 2020;8(4):e10733.

[93] European Medicines Agency. Qualification of novel methodologies for drug development: Guidance to applicants. (2014) Available at: https://www.ema.europa.eu/en/ documents/regulatory-procedural-guideline/qualificationnovel-methodologies-drug-development-guidance-applica nts_en.pdf (Accessed October 25, 2021).

[94] Innovative Medicines Initiative. Report from the IMIEMA-FDA Regulatory Science Summit - Collaborative research through public-private partnership in support to advancing regulatory science. (2017) Available at: https:// www.imi.europa.eu/sites/default/files/uploads/documents/ news-events/press-releases/RegulatorySummit2017_report .pdf. (Accessed October 25, 2021).

[95] Innovative Medicines Initiative. Raising awareness of regulatory requirements. A guidance tool for researchers. (2015) Available at: https://www.imi.europa.eu/sites/default/files/ uploads/documents/apply-for-funding/call-documents/ imi2/RegulatoryRequirementsGuide.pdf (Accessed October 25, 2021). 